\title{
INTEGRAL SURFACES OF PAIRS OF PARTIAL DIFFERENTIAL EQUATIONS OF THE THIRD ORDER*
}

\author{
BY
}

ERNEST P. LANE

\section{INTRODUCTION}

There is already in existence an extensive theory $\dagger$ of the integral surfaces of linear homogeneous partial differential equations of the second order. It is known that, by suitable choice of the parametric curves on an integral surface of a single equation of this kind, the equation can be reduced to an equation of Laplace when the surface sustains a conjugate net, or else to an equation of the parabolic type when the surface sustains a one-parameter family of asymptotic curves. Moreover, an integral surface of two essentially distinct equations of the second order is either a developable surface or else is a nondevelopable surface immersed in ordinary space $S_{3}$. Finally, an integral surface of three such equations is a plane.

It is natural to seek to develop a theory of integral surfaces of equations of the third order analogous to the theory briefly indicated in the foregoing paragraph. Some work in this direction $\ddagger$ has already been done. On an integral surface $S$ of a single equation of the third order of the form

$$
A x_{u u u}+3 B x_{u u v}+3 C x_{u v v}+D x_{v v v}+T=0 \text {, }
$$

where not all of $A, B, C, D$ are zero and $T$ denotes, as throughout this paper, a linear combination of $x, x_{u}, x_{v}, x_{u u}, x_{u v}, x_{v v}$, there exists a covariantly defined triple $\S$ of one-parameter families of curves whose differential equation is

$$
D d u^{3}-3 C d u^{2} d v+3 B d u d v^{2}-A d v^{3}=0 .
$$

In fact, it will be shown in the next paragraph that at each point $P_{x}$ of the surface $S$ the triple of directions of these curves is the unique triple which is

* Presented to the Society, December 30, 1929; received by the editors November 20, 1929.

† Segre, Su una classe di superficie degl'iperspazi legata colle equazioni lineari alle derivate parziali di $2^{\circ}$ ordine, Atti della Reale Accademia delle Scienze di Torino, vol. 42 (1907), p. 1047.

† Bompiani, Determinazione delle superficie integrali d'un sistema di equazioni a derivate parziali lineari ed omogenee, Rendiconti del Reale Istituto Lombardo di Scienze e Lettere, vol. 52 (1919), p. 626.

\& Segre, loc. cit., p. 1079. 
apolar to every triple of directions of curves of section of $S$ and a suitably restricted variable hyperplane through $P_{x}$. There are different possibilities as to the coincidences of the families of curves (2), and as to the corresponding canonical forms that can be obtained for equation (1) by appropriate choices of the parametric curves on the surface $S$.

We shall now explain more precisely Segre's geometrical definition of the covariant triple: if one-parameter families of curves represented analytically by equation (2). Let us consider a point $P_{x}$ on a surface $S$, and indicate as usual by the symbol $S(2,0)$ the linear space of least dimensions that contains the osculating plane at the point $P_{x}$ of every curve on the surface $S$ that passes through $P_{x}$. It is well known that this space is determined by the six points $x, x_{u}, x_{v}, x_{u u}, x_{u v}, x_{v v}$, so that it is ordinarily a space of five dimensions. Any hyperplane with coördinates $\xi^{(1)}, \cdots, \xi^{(n+1)}$, which contains the space $S(2,0)$, is restricted by the conditions

(2a) $\sum \xi x=0, \sum \xi x_{u}=0, \sum \xi x_{v}=0, \sum \xi x_{u u}=0, \sum \xi x_{u v}=0, \sum \xi x_{v v}=0$,

the summation ranging from 1 to $n+1$. This hyperplane cuts the surface $S$ in a curve with a triple point at $P_{x}$, the directions of the triple-point tangents being given by the equation

$$
\sum \xi x_{u u u} d u^{3}+3 \sum \xi x_{u u v} d u^{2} d v+3 \sum \xi x_{u v v} d u d v^{2}+\sum \xi x_{v v v} d v^{3}=0 .
$$

But by means of the conditions (2a) we find from equation (1) the relation

$$
A \sum \xi x_{u u u}+3 B \sum \xi x_{u u v}+3 C \sum \xi x_{u v v}+D \sum \xi x_{v v v}=0,
$$

which says that at the point $P_{x}$ the triple of directions given by equation (2) is the unique triple of directions which is apolar to every triple of directions given by equation (2b). Geometrically, if three directions (2b) coincide at all, they coincide in one of the three directions (2). Thus the origin of equation (2) and its geometrical significance are made clearer.

This paper is concerned with integral surfaces of a pair of linear homogeneous partial differential equations of the third order. The first problem is to reduce the pair of equations to canonical forms. It is found that there are six such canonical forms corresponding to six projectively distinct geometrically described classes of integral surfaces. The basis of the classification lies in the discussion of the singular triples of covariant families of curves in the next section, where the first canonical form is obtained. In the third section the other five canonical forms are reached. In the fourth section the integrability conditions are briefly discussed, and some geometric results are obtained. 
II. THE FIRST CANONICAL FORM OF THE DIFFERENTIAL EQUATIONS

In this section singular covariant triples of families of curves are defined on an integral surface of a pair of differential equations of the third order, and a beginning is made of the reduction of the pair of equations to canonical forms, the first canonical form being obtained.

Let us consider in a linear projective space of $n$ dimensions $S_{n}$ an integral surface $S$ of a pair of differential equations of the form

$$
\begin{aligned}
A x_{u u u}+3 B x_{u u v}+3 C x_{u v v}+D x_{v v v}+T & =0, \\
A^{\prime} x_{u u u}+3 B^{\prime} x_{u u v}+3 C^{\prime} x_{u v v}+D^{\prime} x_{v v v}+T^{\prime} & =0 .
\end{aligned}
$$

Let us suppose that these equations are essentially distinct in the sense that the respective coefficients of the third derivatives are not proportional. Let us further suppose that the surface $S$ is not an integral surface of more than two essentially distinct equations of the third order, and is not an integral surface of any equation of the second order.

The surface $S$ is an integral surface of a pencil* of differential equations. A general one of these can be written, on multiplying the first of equations (3) by a function $\lambda$ and the second by $\mu$, in the form

$$
\alpha x_{u u u}+3 \beta x_{u u v}+3 \gamma x_{u v v}+\delta x_{v v v}+T=0,
$$

where

(5) $\alpha=\lambda A+\mu A^{\prime}, \quad \beta=\lambda B+\mu B^{\prime}, \quad \gamma=\lambda C+\mu C^{\prime}, \quad \delta=\lambda D+\mu D^{\prime}$.

On the surface $S$ there exists a system of covariant triples of one-parameter families of curves. This system depends linearly on the ratio $\lambda / \mu$, the differential equation of a general triple being, after the analogy of equaton (2),

$$
\delta d u^{3}-3 \gamma d u^{2} d v+3 \beta d u d v^{2}-\alpha d v^{3}=0 .
$$

Such of these triples as consist of two coincident families and one distinct family will be called singular triples of the first kind; the two coincident families may be referred to as a double family, and the distinct family as a single family. Such of the triples as consist of three coincident families will be called singular triples of the second kind; the three coincident families may be referred to as a three-fold family. Those values of $\lambda / \mu$ which correspond to singular triples are roots of the quartic equation obtained by setting equal to zero the discriminant of the binary cubic form in equation (6), namely,

$$
(\alpha \delta-\beta \gamma)^{2}-4\left(\alpha \gamma-\beta^{2}\right)\left(\beta \delta-\gamma^{2}\right)=0 .
$$

\footnotetext{
* Bompiani, loc. cit., p. 630 .
} 
Furthermore, those values of $\lambda / \mu$ which correspond to singular triples of the second kind are roots of the three quadratic equations

$$
\alpha \delta-\beta \gamma=0, \quad \alpha \gamma-\beta^{2}=0, \quad \beta \delta-\gamma^{2}=0 .
$$

The differential equations (4) that correspond to values of $\lambda / \mu$ satisfying equation (7) may be called singular equations.

Since every algebraic equation has at least one root, it follows that on every integral surface $S$ of system (3) there is at least one singular triple of families of curves. Let us suppose in the remainder of this section that the surface $S$ sustains no singular triple of the first kind. Then there exists on $S$ at least one singular triple of the second kind. Let us suppose that we have made a transformation of parameters so that the curves of this singular triple have become the $u$-curves. Now equation (6) shows that there exists a value of the ratio $\lambda / \mu$ such that $\beta=\gamma=\delta=0, \alpha \neq 0$. Let us take as the second of equations (3) the equation (4) that corresponds to this value of $\lambda / \mu$. Thus we see that the fundamental differential equations can be written in the form

$$
\begin{gathered}
3 B x_{u u v}+3 C x_{u v v}+D x_{v v v}+T=0, \\
x_{u u u}+T^{\prime}=0 .
\end{gathered}
$$

Equations (5) now become

$$
\alpha=\mu, \beta=\lambda B, \gamma=\lambda C, \delta=\lambda D,
$$

and therefore equation ( 7$)$ reduces to

$$
\lambda^{2}\left[B^{2}\left(4 B D-3 C^{2}\right) \lambda^{2}+2 C\left(2 C^{2}-3 B D\right) \lambda \mu+D^{2} \mu^{2}\right]=0,
$$

while equations (8) become

$$
\lambda(\mu D-\lambda B C)=0, \quad \lambda\left(\mu C-\lambda B^{2}\right)=0, \quad \lambda^{2}\left(B D-C^{2}\right)=0 .
$$

System (9) can be still further simplified. Every solution of equation (11) must, under the hypotheses that have been made, also satisfy equations (12). It is obvious that the solution $\lambda=0$ does so. Now it can be shown that $D \neq 0$ by showing that the supposition $D=0$ leads to a contradiction; the details of this indirect proof need not be elaborated here beyond saying that two cases may be considered according as $C=0$ or $C \neq 0$. Let us take $D=1$; the second factor of the left member of equation (11) vanishes only if $\lambda \neq 0$. The third of equations (12) gives $B=C^{2}$, and equation (11) now becomes

$$
\lambda^{2}\left(C^{3} \lambda-\mu\right)^{2}=0 \text {, }
$$

equations (12) being all satisfied by the roots of this equation. Therefore equations (9) can be written in the form 


$$
\begin{gathered}
3 C^{2} x_{u u v}+3 C x_{u v v}+x_{v v v}+T=0, \\
x_{u u u}+T^{\prime}=0 .
\end{gathered}
$$

On an integral surface $S$ of this system the covariant triples have the differential equation

$$
\lambda(d u-C d v)^{3}+\left(C^{3} \lambda-\mu\right) d v^{3}=0 .
$$

Of these triples the only ones that are singular are the one for which $\lambda=0$ and the one for which $C^{3} \lambda-\mu=0$.

In order to make the final step in the reduction of system (3) to the first canonical form, let us observe that the v-curves on the surface $S$ have not yet been specialized. Let us suppose that they are now chosen as the curves of the singular triple for which $C^{3} \lambda-\mu=0$. This choice amounts to making $C=0$ in equations (13). Our results may be summarized as follows.

If the integral surfaces of system (3) have on them no singular triples of the first kind, then this system can be reduced to the canonical form

$$
x_{v v v}+T=0, \quad x_{u u u}+T^{\prime}=0,
$$

for which the differential equation of a general covariant triple is

$$
\lambda d u^{3}-\mu d v^{3}=0,
$$

and the singular triples are given $b y \lambda^{2} \mu^{2}=0$.

Incidentally it has been shown that there are just two distinct singular triples of the second kind on an integral surface $S$ of a system of the form (3) which has on it no singular triple of the first kind.

\section{Completion of the REDUCTION to CANONICAL forms}

In this section the reduction of system (3) to canonical forms is continued and completed. Having already considered and disposed of the case in which an integral surface of system (3) has on it no singular triple of the first kind, we may now suppose that an integral surface $S$ has on it at least one singular triple of the first kind. Let us suppose that the parametric curves on the surface $S$ have been so chosen that the double family of a singular triple of the first kind consists of the $u$-curves and the single family consists of the $v$-curves. Now equation (6) shows that there exists a value of the ratio $\lambda / \mu$ such that $\alpha=\gamma=\delta=0, \beta \neq 0$. Let us take the equation (4) that corresponds to this value of $\lambda / \mu$ as the second of equations (3). Thus we see that we can use as our fundamental equations the system

$$
\begin{gathered}
A x_{u u u}+3 C x_{u v v}+D x_{v v v}+T=0, \\
3 x_{u u v}+T^{\prime}=0 .
\end{gathered}
$$


Equations (5) now become

$$
\alpha=\lambda A, \quad \beta=\mu, \quad \gamma=\lambda C, \quad \delta=\lambda D,
$$

and therefore equation (7) reduces to

$$
\lambda\left[A\left(A D^{2}+4 C^{3}\right) \lambda^{3}-6 A C D \lambda^{2} \mu-3 C^{2} \lambda \mu^{2}+4 D \mu^{3}\right]=0,
$$

while equations (8) become

$$
\lambda(\lambda A D-\mu C)=0, \quad \lambda^{2} A C-\mu^{2}=0, \quad \lambda\left(\mu D-\lambda C^{2}\right)=0 .
$$

It can be shown by an indirect proof that there is another singular triple on the surface $S$ besides the triple $d u d v^{2}=0$. In fact, if there were no other singular triple then $\lambda=0$ would be a quadruple root of equation (19). Consequently we should have $C=D=0, A \neq 0$. Taking $A=1$ we should find from equation (6) that every triple of the system

$$
3 \mu d u d v^{2}-\lambda d v^{3}=0
$$

is singular, and a contradiction would thus be reached.

System (17) can be reduced still more by taking as the first equation therein another singular equation. Then $\mu=0$ is a solution of equation (19). It follows that

$$
A\left(A D^{2}+4 C^{3}\right)=0 .
$$

Three possibilities present themselves:

1.

$$
\begin{array}{ll}
A=0, & C \neq 0 . \\
A=0, & C=0, \quad D \neq 0 . \\
A \neq 0, & A D^{2}+4 C^{3}=0 .
\end{array}
$$

2 .

3.

Let us for the moment suppose that the singular triple corresponding to $\mu=0$ is of the second kind. Since $\mu=0$ must be a solution of equations (20) it follows that $C=A D=0$, and two cases appear. First, if $C=A=0, D=1$, system (17) takes the canonical form

$$
x_{v v v}+T=0, \quad 3 x_{u u v}+T^{\prime}=0,
$$

for which the differential equation of the covariant triples is

$$
\lambda d u^{3}+3 \mu d u d v^{2}=0,
$$

and the singular triples are given by $\lambda \mu^{3}=0$. Therefore on an integral surface of system (22) there is one singular triple of the first kind and one of the second kind, so related that the curves of the three-fold family of the second triple are the 
curves of the single family of the first triple. In the second case, when $C=D=0$, $A=1$, the canonical form of system (17) is

$$
x_{u u u}+T=0, \quad 3 x_{u u v}+T^{\prime}=0 .
$$

The differential equation of the covariant triples is

$$
3 \mu d u d v^{2}-\lambda d v^{3}=0,
$$

and the singular triples are indeterminate, every covariant triple being singular. There are a one-parameter family of singular triples of the first kind and just one singular triple of the second kind on an integral surface of system (23).

Let us finally suppose that the singular triple corresponding to $\mu=0$ is of the first kind. The possibilities not yet considered are the following:

1.

$$
A=0, \quad C \neq 0 .
$$

2 .

$$
A C D \neq 0, \quad A D^{2}+4 C^{3}=0 \text {. }
$$

It is necessary to consider two subcases in connection with the first possibility, according as $D=0$ or $D \neq 0$. In the first subcase, when $A=D=0$, $C=1$, the canonical form of system (17) is

$$
3 x_{u v v}+T=0, \quad 3 x_{u u v}+T^{\prime}=0 .
$$

The differential equation of the covariant triples is

$$
\lambda d u^{2} d v-\mu d u d v^{2}=0,
$$

and the singular triples are given by $\lambda^{2} \mu^{2}=0$. On an integral surface of system (24) there are just two singular triples; both are of the first kind; the single family of each triple is the double family of the other. In the second subcase, when $A=$ $0, C=1, D \neq 0$, the canonical form of system (17) is

$$
3 x_{u v v}+D x_{v v v}+T=0,3 x_{u u v}+T^{\prime}=0 .
$$

The differential equation of the covariant triples is

$$
\lambda D d u^{3}-3 \lambda d u^{2} d v+3 \mu d u d v^{2}=0,
$$

and the singular triples are given by $\lambda \mu^{2}(3 \lambda-4 D \mu)=0$, their differential equations being, respectively,

$$
d u d v^{2}=0, \quad d u^{2}(D d u-3 d v)=0, \quad d u(2 D d u-3 d v)^{2}=0 .
$$

On an integral surface of system (25) there are just three singular triples; all are of the first kind; two of these triples have the same single family, which is also the double family of the other triple. 
For the last possibility, when $A C \neq 0, D=1, A=-4 C^{3}$, the canonical form of system (17) is

$$
-4 C^{3} x_{u u u}+3 C x_{u v v}+x_{v v v}+T=0, \quad 3 x_{u u v}+T^{\prime}=0 .
$$

The differential equation of the covariant triples is

$$
\lambda d u^{3}-3 \lambda C d u^{2} d v+3 \mu d u d v^{2}+4 \lambda C^{3} d v^{3}=0,
$$

and the singular triples are given by $\lambda \mu=0$ and

$$
\mu / \lambda=\left[3 \pm 5(-15)^{1 / 2}\right] C^{2} / 8,
$$

their differential equations being, respectively,

$$
\begin{gathered}
d u d v^{2}=0, \quad(d u+C d v)(d u-2 C d v)^{2}=0, \\
\left\{4 d u+\left[1 \mp(-15)^{1 / 2}\right] C d v\right\}^{2}\left\{2 d u+\left[-7 \pm(-15)^{1 / 2}\right] C d v\right\}=0 .
\end{gathered}
$$

On an integral suface of system (26) there are four singular triples; all are of the first kind; there is no family common to any two of the singular triples.

Thus the reduction of system (3) to canonical forms is completed. There are six of these forms written in equations (15), (22), (23), (24), (25), (26). Each canonical form has been characterized by a different projective geometric description of its integral surfaces, as may be clearly seen in the following way. Let us associate with each canonical form a symbol $(p, q)$ to indicate that on an integral surface there are $p$ singular triples of the first kind and $q$ of the second kind. Then the six canonical forms in the order designated above have, respectively, the symbols

$$
(0,2),(1,1),(\infty, 1),(2,0),(3,0),(4,0),
$$

which are obviously all distinct.

It seems appropriate to say here precisely what is the relation between the present paper and the paper of Bompiani cited earlier. On page 630 of his paper Bompiani attacks the problem of reducing a pair of third-order equations to canonical forms, using apparently the same point of view that we have adopted. On page 631 , he erroneously states* that "since the discriminant itself is of the third degree in the coefficients, therefore in the parameter, there will exist in general three equations in the pencil having the desired property." The words third and three should obviously be fourth and four, respectively. He obtains our equations (17), and then, appealing to some general theory developed earlier in his paper, arrives at two canonical forms which do not differ essentially from our canonical forms (23) and (24). $\mathrm{He}$ does not discover our four other canonical forms. He does not develop a

\footnotetext{
* Author's translation.
} 
theory based on our canonical form (23), but confines his further studies to our canonical form (24), obtaining extremely interesting results to which we shall refer again in the next section.

\section{DEDUCTIONS FROM THE CANONICAL FORMS}

For each of the six canonical forms of the differential equations under consideration there are certain integrability conditions that must be satisfied by the coefficients. The complete calculation of all of these would extend this paper beyond the bounds assigned for it. So the integrability conditions will only be discussed briefly in the first four cases here. The reader is referred for further details of these cases and the theory of the last two cases to the work of one of my students, Mr. C. F. Bowles, who is following up this study in his Chicago doctoral dissertation. Moreover, there are certain geometrical consequences easily deducible from the differential equations and the integrability conditions. Some of these geometrical results will also claim our attention in this section.

It is convenient to rewrite equations (15) in order to give explicit expressions for the terms of the second and lower orders:

$$
\begin{aligned}
& x_{u u u}=a x_{u u}+h x_{u v}+b x_{v v}+l x_{u}+m x_{v}+d x, \\
& x_{v v v}=a^{\prime} x_{u u}+h^{\prime} x_{u v}+b^{\prime} x_{v v}+l^{\prime} x_{u}+m^{\prime} x_{v}+d^{\prime} x .
\end{aligned}
$$

The form of these equations readily yields a geometric theorem, the statement of which is facilitated by Bompiani's definition of the osculating space $S(k, 0)$ at a point $P_{x}$ of a surface. This space $S(k, 0)$ is the linear space of least dimensions containing the osculating linear space of $k$ dimensions $S_{k}$ at the point $P_{x}$ of every curve on the surface through $P_{x}$. It follows from this definition that the space $S(k, 0)$ is determined by $x$ and the derivatives of $x$ up to and including those of order $k$, so that the space $S(k, 0)$ is ordinarily of $\frac{1}{2} k$ $\cdot(k+3)$ dimensions. In particular, as was pointed out in Section I, the space $S(2,0)$ at a general point of one of our surfaces is the space $S_{5}$ of the points

$$
x, x_{u}, x_{v}, x_{u u}, x_{u v}, x_{v v} .
$$

Now the form of the first of equations (28) shows* that the osculating space $S_{3}$ at any point of a u-curve on an integral surface of system (28) lies in the space $S(2,0)$ of the surface at the point. The second of $(28)$ gives a similar theorem regarding any $v$-curve on the surface.

We may be permitted to indicate here a couple of well known facts. The space $S(3,0)$ at a point of a surface is ordinarily a space $S_{9}$. But at a point

* Bompiani, loc. cit., p. 628. 
of an integral surface of two equations of the third order, such as we have under consideration in this paper, the space $S(3,0)$ is a space $S_{7}$, since the two equations are two linear relations connecting the ten points determining the space $S(3,0)$.

Let us consider the integrability conditions of equations (28). By means of the processes of differentiation and substitution it is possible to express each of the derivatives $x_{u u u}, x_{u u v v}, x_{u v v v}, x_{v v v v}$ uniquely as a linear combination of $x_{u u v}, x_{u v v}$ and derivatives of lower orders including $x$ undifferentiated. Then it is possible to express each of the fifth derivatives of $x$ as a linear combination of the same derivatives and $x_{u u v v}$. Consequently the derivative $x_{u u u v v v}$ can be calculated in two ways as a linear combination of $x_{u u v v}$, $x_{u u v}, x_{u v v}$ and derivatives of lower orders. When these two expressions are equated, two cases arise, according as $a_{v} \neq b_{u}{ }^{\prime}$ or $a_{v}=b_{u}{ }^{\prime}$. In the first case, when $a_{v} \neq b_{u}{ }^{\prime}$, we obtain a differential equation of the fourth order in which the only fourth derivative is $x_{u u v v}$. Therefore, if $a_{v} \neq b_{u}^{\prime}$, every fourth derivative of $x$ can be expressed as a linear combination of $x_{u u v}, x_{u v v}$ and derivatives of lower orders, and an integral surface of system (28) is immersed in a space $S_{7}$. The integrability conditions are to be found from the fact that each of the derivatives $x_{u u v v v}$ and $x_{u u v v v}$ can be calculated in two ways. In the second case, when $a_{v}=b_{u}{ }^{\prime}$, a differential equation of the third order results when the two expressions for $x_{u u v v v}$ are equated. In virtue of assumptions already made, this equation must be an identity so that eight additional integrability conditions are found. Therefore, if $a_{v}=b_{u}{ }^{\prime}$, every fifth derivative of $x$ can be expressed as a linear combination of $x_{u u v v}, x_{u u v}, x_{u v v}$ and derivatives of lower orders, and an integral surface of system (28) is immersed in a space $S_{8}$.

Let us rewrite equations (22) in the form

$$
\begin{aligned}
& x_{u u v}=a x_{u u}+h x_{u v}+b x_{v v}+l x_{u}+m x_{v}+d x, \\
& x_{v v v}=a^{\prime} x_{u u}+h^{\prime} x_{u v}+b^{\prime} x_{u v}+l^{\prime} x_{u}+m^{\prime} x_{v}+d^{\prime} x .
\end{aligned}
$$

The second of these equations tells us again that the osculating space $S_{3}$ at each point of a $v$-curve is the space $S(2,0)$ at the point.

In order to calculate the integrability conditions of system (30) one would first of all express $x_{u u u v}, x_{u u v v}, x_{u v v v}, x_{v v v v}$ as linear combinations of $x_{u u u}$, $x_{u v v}$ and derivatives of lower orders. Then $x_{u u u v v}, x_{u u v v}, x_{u u v v v}, x_{u v v v v}$, $x_{v v v v}$ can be expressed as linear combinations of $x_{u u u u}, x_{u u u}, x_{u v v}$ and derivatives of lower orders; moreover, $x_{u u v v v}$ can be so expressed in two ways, the coefficient of $x_{\text {uuu }}$ being zero in one expression and being $a^{\prime}$ in the other. When these two expressions are equated, two cases arise, according as $a^{\prime}$ $\neq 0$, or $a^{\prime}=0$. In the first case, when $a^{\prime} \neq 0$, we obtain a differential equation 
of the fourth order in which the only fourth derivative is $x_{u u u u}$. Therefore, if $a^{\prime} \neq 0$, every fourth derivative of $x$ can be expressed as a linear combination of $x_{u u u}, x_{u v v}$ and derivatives of lower orders, and an integral surface of system (30) is immersed in a space $S_{7}$. The integrability conditions are to be found from two expressions for $x_{\text {uuuuv }}$. In the second case, when $a^{\prime}=0$, a differential equation of the third order results when the two expressions for $x_{u u v v v}$ are equated, which must be an identity, so that eight other integrability conditions are found, but the space of immersion of an integral surface of system (30) is not restricted to be other than a space $S_{n}$, with $n \geqq 7$.

Rewriting equations (23) in the form

$$
\begin{aligned}
& x_{u u u}=a x_{u u}+h x_{u v}+b x_{v v}+l x_{u}+m x_{v}+d x, \\
& x_{u u v}=a^{\prime} x_{u u}+h^{\prime} x_{u v}+b^{\prime} x_{v v}+l^{\prime} x_{u}+m^{\prime} x_{v}+d^{\prime} x
\end{aligned}
$$

we observe that the osculating space $S_{3}$ at each point of a $u$-curve lies in the space $S(2,0)$ at the point. The derivatives $x_{u u u u}, x_{u u v v}$ can be expressed uniquely as linear combinations of $x_{u v v}, x_{v v v}$ and derivatives of lower orders, whereas $x_{u u u v}$ can be so expressed in two ways. Equating the two expressions for $x_{u u v v}$, we obtain the following eight integrability conditions:

$b=0, h=b^{\prime}, a_{v}=a_{u}^{\prime}+a^{\prime} h^{\prime}+l^{\prime}, \quad h_{v}+a h^{\prime}+l=h_{u}^{\prime}+a^{\prime} h+h^{\prime 2}+m^{\prime}$,

$$
\begin{gathered}
a b^{\prime}+m=b_{u}^{\prime}+h^{\prime} b^{\prime}, \quad l_{v}+a l^{\prime}=l_{u}^{\prime}+a^{\prime} l+h^{\prime} l^{\prime}+d^{\prime}, \\
m_{v}+a m^{\prime}+d=m_{u}^{\prime}+a^{\prime} m+h^{\prime} m^{\prime}, \\
d_{v}+a d^{\prime}=d_{u}^{\prime}+a^{\prime} d+h^{\prime} d^{\prime} .
\end{gathered}
$$

It is not possible to express, by the processes of differentiation and substitution, all derivatives of the fifth or any higher order in terms of derivatives of lower order. Therefore an integral surface of system (31) may lie in any space $S_{n}$ with $n \geqq 7$.

Equations (24) may be rewritten in the form

$$
\begin{aligned}
& x_{u u v}=a x_{u u}+h x_{u v}+b x_{v v}+l x_{u}+m x_{v}+d x, \\
& x_{u v v}=a^{\prime} \cdot x_{u u}+h^{\prime} x_{u v}+b^{\prime} x_{v v}+l^{\prime} x_{u}+m^{\prime} x_{v}+d^{\prime} x .
\end{aligned}
$$

This system of equations together with the integrability conditions and the integral surfaces thereof have been studied by Bompiani, ${ }^{*}$ who has considered the analogy between this system of two equations of the third order and the Laplace equation of the second order, and has studied particularly transformations of this system analogous to the well known transformations of Laplace for the equation that bears his name. Equating the two expressions

* Bompiani, loc. cit., pp. 632-636. 
for $x_{u u v v}$ obtainable for equations (33) leads to eight integrability conditions of which two are $a^{\prime}=b=0$. The space in which the integral surfaces lie is any space $S_{n}$ with $n \geqq 7$.

There is an interesting interpretation of the conditions $a^{\prime}=b=0$ whtch seems to have escaped notice hitherto. Let us consider the ruled surface $R_{u}$ of $u$-tangents constructed at points of a $v$-curve. Let us consider the linear space of least dimensions which contains the osculating planes, at points of a generator $x x_{u}$ of the surface $R_{u}$, of every curve on $R_{u}$ that intersects the generator $x x_{u}$. This space is determined by the points $x, x_{v}, x_{v v}, x_{u}, x_{u v}$, $x_{u v v}$ and is ordinarily a space $S_{5}$ called the tangent space $S_{5}$ of the ruled surface $R_{u}$ along the generator $x x_{u}$. But the last of equations (33) shows that for an integral surface of these equations this space is a space $S_{4}$. Therefore, for an integral surface of equations (33), the osculating planes, at points on a generator $x x_{u}$ on a ruled surface $R_{u}$, of all the curves on $R_{u}$ that intersect the line $x x_{u}$ lie in a space $S_{4}$. There is a symmetric theorem with $u$ and $v$ interchanged.

The analogy which interested Bompiani is further illuminated by the following consideration. One of my students, Mr. G. D. Gore, has remarked to me that if two surfaces have their points in one-to-one correspondence so that their tangent planes at corresponding points intersect in straight lines, then each surface is an integral surface of a system of equations of the type (33), or else of an equation of Laplace, provided that the parameters of the surfaces are suitably chosen.

University of Chicago, Chicago, Ill. 